\title{
Avaliação da dieta de gestantes com sobrepeso
}

\section{Evaluation of diet of overweight pregnant women}

Elizabeth do NASCIMENTO'

Sônia Buongermino de SOUZA²

RESUMO

O objetivo deste estudo foi avaliar o consumo alimentar e verificar a adequação de calorias e de alguns nutrientes em gestantes com sobrepeso pré-gestacional. Estudou-se uma amostra de 110 gestantes que freqüentavam ambulatório de assistência pré-natal na cidade de São Paulo. A alimentação foi verificada pelo método de inquérito recordatório de 24 horas. A proporção dos macronutrientes foi de $55 \%$ de carboidratos, $16 \%$ de proteínas e $29 \%$ de lipídios. A média de energia da dieta foi de $1736 \mathrm{kcal}$. Entre os micronutrientes analisados, apresentaram consumo inadequado: o cálcio, com aproximadamente $50 \%$ de adequação; o ferro, com 33\%; e o folato, com $43 \%$. A dieta mostrou-se satisfatória em energia e insuficiente em relação ao cálcio, ao ferro e ao folato. Estes dados permitem supor a existência de deficiências nutricionais específicas no grupo estudado.

Termos de indexação: gravidez, ganho de peso, alimentação, dieta.

\section{A B S TR A C T}

The objective of this study was to verify the adequacy of calories and some nutrients intake in pregnant women with pregestational overweight. A sample of 110 pregnant women, attending a prenatal assistance ambulatory of the city of São Paulo (Brazil), was evaluated. The diet was investigated by the 24-hour recall method. The proportion of macronutrients was $55 \%$ of carbohydrates, $16 \%$ of proteins and $29 \%$ of lipids. The median caloric consumption was $1736 \mathrm{cal}$. The intake of some micronutrients was fitting the recommendations in about 50\% for calcium, 33\% for iron and $43 \%$ for folate. The diet calories were satisfactory, but the amounts of calcium, iron and folate were insufficient. These data permit a presumption of the presence of specific nutritional deficiencies in the studied group.

Index terms: pregnancy, weight gain, feed, diet.

\footnotetext{
1 Departamento de Nutrição, Centro de Ciências da Saúde, Universidade Federal de Pernambuco.

2 Departamento de Nutrição, Faculdade de Saúde Pública, Universidade de São Paulo. Av. Dr. Arnaldo, 715, 01246-904, São Paulo, SP, Brasil. Correspondência para/Correspondence to: S.B.SOUZA. E-mail: buonger@usp.br
} 


\section{NTRODUÇ Ã O}

A alimentação tem papel relevante para a saúde dos indivíduos, principalmente nas etapas da vida caracterizadas pelo aumento da demanda de energia e de nutrientes, como a gestação. Neste período ocorrem intenso e peculiar processo de formação de tecidos e grandes transformações orgânicas durante um curto espaço de tempo (King \&Weininger, 1991; Guthrie \& Picciano, 1995).

Atualmente, com o aumento do sobrepeso na população brasileira, sobretudo em mulheres, tornam-se necessários o acompanhamento mais eficiente do ganho de peso durante a gestação e o atendimento nutricional não apenas para as gestantes com baixo peso, mas, também, para aquelas com sobrepeso pré-gestacional e risco de ganho excessivo de peso durante a gravidez (Engstrom \& Anjos, 1996).

Apesar da forte correlação entre as mudanças de peso da mãe e o da criança, segundo Susser (1991), em termos de peso ao nascer, a nutrição e a dieta materna merecem mais atenção que o ganho de peso materno por si só.

O total de energia da dieta de gestantes com sobrepeso prévio ou ganho de peso excessivo durante a gestação deve ser menor em relação ao daquelas com peso prévio ou gestacional normal; porém, as quantidades adicionais de proteínas e outros nutrientes são as mesmas para os dois grupos (Bourne, 1987; Neuhouser, 1996).

Alguns nutrientes devem ser analisados quando se estuda a alimentação de gestantes, em virtude de serem os que têm maior probabilidade de consumo inadequado, pelo fato de não serem amplamente distribuídos nos alimentos e/ou por suas recomendações serem percentualmente muito maiores em comparação com os demais. Entre estes encontram-se cálcio, fósforo, retinol, vitamina C, folato e ferro.

Considerando os aspectos abordados, o objetivo do presente trabalho foi verificar o valor energético e a adequação nutricional da ingestão dietética de mulheres grávidas, com sobrepeso prévio ou adquirido durante a gestação, obser- vando-se a ocorrência de consumo adequado de nutrientes.

\section{CASUISTICAE MÉTODOS}

Foi realizado um estudo transversal, de caráter descritivo, com mulheres em diferentes etapas da gestação. Verificou-se a ingestão energética e de alguns nutrientes relevantes para um satisfatório curso gestacional.

A amostra foi composta de 110 gestantes com sobrepeso pré-gestacional. A seleção seguiu os critérios da World Health... (1995), que considera como sobrepeso o Índice de Massa Corporal (IMC) igual ou maior a $25 \mathrm{~kg} / \mathrm{m}^{2}$. Os dados foram coletados entre dezembro de 1997 e julho de 1998, em ambulatório de pré-natal de instituição beneficente no município de São Paulo.

As medidas de peso foram tomadas em todas as consultas do pré-natal, com as gestantes sem calçados e sem excesso de roupas. A balança utilizada foi do tipo não digital, com subdivisão de $100 \mathrm{~g}$ e carga máxima de $150 \mathrm{~kg}$. A escala para medida da altura era fixa à balança, em haste vertical, com divisão de $0,5 \mathrm{~cm}$ e escala extensiva situada entre 95 e $195 \mathrm{~cm}$. A obtenção da altura, na maioria dos casos, foi realizada na primeira consulta do pré-natal.

O peso pré-gestacional foi obtido através de informação fornecida pela gestante. Apesar de esse valor estar sujeito a erros de memória ou de super ou subestimação do peso pré-gravídico, pesquisadores como Steven-Simon et al. (1992) verificaram uma alta correlação entre o referido e o aferido na consulta.

A medida da idade gestacional foi baseada na data da última menstruação, corroborada pela mensuração da altura uterina e pelo exame por ultra-sonografia.

Os dados referentes à ingestão alimentar foram obtidos pela pesquisadora por meio do método de inquérito recordatório de 24 horas, em uma única entrevista. 
Após verificar o valor energético médio da dieta e o consumo médio de nutrientes, os resultados foram comparados com a recomendação do National Research... (1989), e a distribuição percentual de macronutrientes, com a recomendação da Organización Mundial... (1990).

$\mathrm{Na}$ análise estatística foram utilizadas médias, desvios padrão e intervalos com 95\% de confiança. Foram utilizados os programas Virtual Nutri 1.0 e Epi Info versão 6.03. Um termo de consentimento esclarecido foi assinado por todas as gestantes que aceitaram participar do estudo.

\section{RESULTADOS}

As 110 gestantes tinham idade média de $26 \pm 7$ anos), com baixo nível de escolaridade (59\% tinham ensino fundamental incompleto) e com renda per capita média de $\mathrm{R} \$ 230,00$ $( \pm 148,73)$, correspondendo a 1,8 salário mínimo vigente.

Quanto ao número de gestações anteriores, foram obtidos os seguintes percentuais:
$34,5 \%$ estavam na primeira gestação, 33,6\% encontravam-se na segunda e 31,8\% estavam na terceira ou mais.

O IMC médio encontrado entre as gestantes foi de $28,1 \pm 4,2 \mathrm{~kg} / \mathrm{m}^{2}$, indicando a ocorrência de sobrepeso grau I. A Tabela 1 apresenta a distribuição das gestantes de acordo com o índice de massa corpórea pré-gestacional. Segundo se observa, $32 \%$ da amostra tinha sobrepeso igual ou maior que grau ll ou obesidade (IMC $\geq 30 \mathrm{~kg} / \mathrm{m}^{2}$ ).

Com relação ao consumo alimentar, identificou-se uma distribuição percentual de macronutrientes de $16 \%$ de proteínas, $29 \%$ de lipídios e $55 \%$ de carboidratos. Estas proporções foram obtidas a partir dos valores médios de consumo.

A Tabela 2 mostra o percentual de adequação de energia e de nutrientes segundo a recomendação do National Research... (1989). Conforme se verifica, apenas os consumos médios de proteínas, de retinol (vitamina $A$ ) e de vitamina $C$ atingiram e/ou

Tabela 1. Distribuição das gestantes segundo o índice de massa corporal (IMC) pré-gestacional.

\begin{tabular}{lccccc}
\hline $\mathrm{MC}$ & $\mathrm{n}^{\circ}$ & $\%$ & Média & DP* & IC95\%** \\
\hline $25 \longmapsto-30$ & 78 & 70,9 & 26,7 & 1,5 & $26,4-27,0$ \\
$30 \longmapsto 40$ & 29 & 26,4 & 32,9 & 2,0 & $32,2-33,6$ \\
$\geq 40$ & 3 & 2,7 & 45,4 & 3,7 & $41,2-49,6$ \\
\hline Total & 110 & 100,0 & 28,1 & 4,5 & \\
\hline
\end{tabular}

(*) $\mathrm{DP}=$ desvio-padrão

${ }^{(* *)} \mathrm{IC}=$ intervalo de confiança.

Tabela 2. Média, desvio-padrão e percentagem de adequação de energia e do consumo de nutrientes.

\begin{tabular}{lrrrr}
\hline Nutrientes & \multicolumn{1}{c}{ Média } & DP* & RDA** & Adequação(\%) \\
\hline Energia (kcal) & 1736,13 & 630,75 & 2500 & 69,44 \\
Proteínas (g) & 70,38 & 26,36 & 60 & 117,30 \\
Cálcio (mg) & 593,71 & 304,63 & 1200 & 49,48 \\
Fósforo (mg) & 820,05 & 367,38 & 1200 & 68,34 \\
Retinol (mcg) & 1059,40 & 548,62 & 800 & 132,43 \\
Vitamina C $(\mathrm{mg})$ & 110,42 & 105,88 & 70 & 157,74 \\
Folato $(\mathrm{mcg})$ & 170,70 & 114,74 & 400 & 42,68 \\
Ferro $(\mathrm{mg})$ & 9,94 & 4,18 & 30 & 33,13 \\
\hline
\end{tabular}

(*) $\mathrm{DP}=$ desvio-padrão

${ }^{(* *)}$ Recommended Dietary Allowances(National Reserach..., 1989). 
176 | E. NASCIMENTO \& S.B. SOUZA

ultrapassaram as quantidades recomendadas, obtendo-se um percentual de adequação acima de $100 \%$ do padrão adotado.

Separando-se as gestantes em dois grupos de IMC $\left(24,9-29,9 \mathrm{~kg} / \mathrm{m}^{2}\right.$ e $\left.=>30 \mathrm{~kg} / \mathrm{m}^{2}\right)$ e comparando-se as médias de calorias da dieta e ingestão de nutrientes, verificou-se menor consumo médio de retinol entre as mulheres do grupo com maior IMC $(521,93 \mathrm{mcg})$ em relação às do grupo com menor IMC $(1279,90 \mathrm{mcg})$.

\section{DISCUSSÃO}

Pesquisadores como Edwards et al. (1978), Tanaka (1981), Frentzen et al. (1988) e Uchiyama et al. (1993) encontraram em seus estudos com gestantes obesas maior número de multíparas (no mínimo duas gestações anteriores) e de grandes multíparas (quatro ou mais gestações anteriores).

Neste trabalho, apesar de se tratar de gestantes com sobrepeso, observa-se uma maior percentagem de primigestas (mulheres na primeira gravidez). Isso pode ser explicado pelo fato de a amostra ter $17,5 \%$ de mulheres com idade inferior a 20 anos, embora a média encontrada tenha sido de 26 anos. Algumas pesquisas realizadas no Brasil com gestantes com sobrepeso mostraram resultados similares quanto à idade média (Tanaka, 1981; Uchiyama et al., 1993; Diniz \& Sancovski, 1996).

Com relação à distribuição de macronutrientes nas dietas analisadas, verifica-se que seus percentuais estão bem próximos dos recomendados pela Organizacíon Mundial... (1990).

Não obstante, a contribuição energética proveniente dos lipídios (29\% das calorias totais) ficou próxima ao limite máximo recomendado e, como estas gestantes constituem um grupo com excesso de peso, seria prudente a redução do consumo de gorduras. Os fatores relacionados com sobrepeso e/ou obesidade são múltiplos; porém, o papel da dieta não pode ser negligenciado. Existe, ainda, muita discussão sobre qual ou quais nutrientes têm maior influência no aumento de peso. Entretanto, já é consenso que dieta inadequada e grande ingestão de lipídios e de alimentos processados são consideradas condições de risco para o incremento do peso (Abdala et al., 1998).

Entre os micronutrientes analisados, 0 cálcio, o folato e o ferro foram os menos consumidos pela amostra estudada. A média de consumo de folato encontrada nesta pesquisa foi de $170 \mu \mathrm{g} / \mathrm{dia}$, representando $42,5 \%$ da recomendação do National Research... (1989) e estando bem abaixo de médias relatadas por pesquisadores como Mikode \& White (1994), os quais, ao estudarem a dieta de gestantes americanas, verificaram ingestão de 252 a $302 \mu \mathrm{g} /$ dia, valores 1,4 a 1,7 vezes maiores que o encontrado nesta amostra. Donangelo et al. (1996) constataram, em gestantes brasileiras, um consumo médio de folato próximo de $300 \mu \mathrm{g} / \mathrm{dia}$, representando $83 \%$ da quantidade recomendada pelo National Research... (1989).

Um dos problemas para a saúde da criança que pode advir da deficiência de ácido fólico é a má-formação do tubo neural, com possibilidade de subseqüente anemia megaloblástica materna ou do recém-nascido (Shojania 1984; Campbell 1995). Conforme se verificou, a ingestão de 0,8 mg/dia de ácido fólico, durante a gestação, diminui a ocorrência de má-formação de tubo neural, bem como do trato urinário e cardiovascular, e ainda propicia redução de sintomas como náuseas e vômitos nas gestantes e diminuição da incidência de partos prematuros (Czeizel \& Dudàs, 1992).

Em relação ao cálcio, a média de consumo neste estudo foi de $593 \mathrm{mg} / \mathrm{dia}$, valor próximo do obtido no Rio de Janeiro por Donangelo et al. (1996), que foi de $636 \mathrm{mg} / \mathrm{dia}$, porém muito abaixo do relatado por Mikode \& White (1994), 
os quais encontraram nos Estados Unidos ingestão média de 1200 mg/dia. Na gestante, a deficiência deste nutriente está associada à hipertensão gestacional. Além disso, pode haver aumento da mobilização do estoque de cálcio ósseo e da ocorrência de osteoporose pós-menopausa (Yoon et al., 2000).

Quanto ao ferro, a ingestão média foi de $10 \mathrm{mg} / \mathrm{dia}$, valor que expressa apenas um terço das recomendações do National Research... (1989), enquanto Weigel et al. (1991), estudando a dieta de gestantes equatorianas, encontraram consumo médio de $18,5 \mathrm{mg} /$ dia. Bellati et al. (1994) obtiveram média de 12 mg/dia e Mikode \& White (1994) tiveram como resultado 14 e 16 mg/dia no segundo e terceiro trimestres de gestação, respectivamente.

De acordo com duas pesquisas realizadas no Rio de Janeiro, cerca de $40 \%$ das gestantes estudadas apresentavam depleção da reserva de ferro e, portanto, deficiência subclínica do mineral (Donangelo et al., 1989; Trugo et al., 1996). No Brasil a prevalência de anemia ferropriva em gestantes varia de $14 \%$ em São Paulo a 35\% em Pernambuco (Vannucchi et al., 1992).

A recomendação para ingestão de ferro durante a gestação dificilmente é atendida somente com a alimentação, por isso se aconselha a suplementação para todas as gestantes, a partir da $12^{a}$ semana de gravidez (King \& Weininger, 1991).

O total energético da dieta atingiu nível de adequação em torno de $70 \%$, podendo ser considerado satisfatório, visto que as mulheres com sobrepeso não precisam "armazenar" tecido adiposo destinado a suprir o aumento das necessidades energéticas do final da gravidez e do período de lactação (Bourne 1987; Neuhouser, 1996). Além disso, segundo Borghi et al. (1997), o aporte adequado de vitaminas e de minerais só é limitado quando o total energético da dieta não atinge dois terços das recomendações, ou seja, é inferior a 1667 $\mathrm{kcal} / \mathrm{dia}$.

Pode-se supor a ocorrência, no presente estudo, de subnotificação da ingestão de alimentos, pois as gestantes tinham conhecimento de que eram portadoras de sobrepeso e isso poderia levá-las a relatar um consumo menor do que o real.

A relação entre dieta, ganho de peso durante a gestação e peso ao nascer ainda não está totalmente esclarecida. Segundo estudos de pesquisadores como Bellati et al. (1994) e Mikode \& White (1994), distintos consumos energéticos levaram a ganhos de peso das gestantes e pesos ao nascer dos respectivos lactentes muito similares.

Quando as gestantes foram separadas em dois níveis de IMC, não foram verificadas diferenças estatisticamente significativas das calorias totais da dieta e do consumo de nutrientes entre os dois subgrupos. As médias de consumo foram bastante semelhantes, exceto quanto ao retinol, em relação ao qual foi verificado uma menor ingestão no grupo com $I M C \geq 30 \mathrm{~kg} / \mathrm{m}^{2}$. Esta variação é aceitável devido à grande diversidade inter e intraindivíduo de consumo dos alimentos fontes deste nutriente.

Com base nos resultados obtidos, conclui-se que as mulheres estudadas tinham dieta com total energético adequado, com proteínas e lipídios em limite superior ao da recomendação da Organización Mundial... (1990) e com quanti-dades de ferro e folato insuficientes (National Research... 1989). Diante disso, supõe-se estarem as gestantes com sobrepeso, assim como as normais, predispostas a carências nutricionais específicas. Elas necessitam, portanto, de atendimento nutricional, visando o controle do ganho de peso e o adequado aporte de nutrientes. 
REFERENCIAS

BIBLIOGRAFICAS

ABDALA, B.C., DEL RIO, F.V., KAIN, B.J. Obesidade: un desafio pendiente en Chile. Revista de Medicina Chilena, Santiago, v.126, n.8, p.1001-1109, 1998.

BELLATI, U., POMPA, P., LIBERATI, M. Valutazione analitica di una dieta "mediterranea" in gravidanza. Minerva Ginecologica, Torino, v.46, n.4, p.183-187, 1994.

BORGHI, R., BAXTER, Y.C., GALLETA, M.A., MACULEVICIUS, J., ZUGAIB, M. Mudanças no comportamento alimentar em gestantes adolescentes. Revista de Ginecologia e Obstetrícia, São Paulo, v.8, n.1, p.20-22, 1997.

BOURNE, H.G. Energy: nutrition of women. Basel : KARGER, 1987. 52p.

CAMPBELL, B.A. Megaloblastic anemia in pregnancy. Clinical Obstetrics and Gynecology, Philadelphia, v.38, n.3, p.455-462, 1995.

CZEIZEL, A.E., DUDÀS, I. Prevention of the first occurrence of neural tube defects by periconceptional vitamin supplementation. New England Journal of Medicine, London, v.327, n.2, p.1832-1835, 1992.

DINIZ, L.E.V., SANCOVSKI, M. Efeitos da dieta com restrição calórica moderada sobre o binômio obesidade e síndrome hipertensiva na gravidez. Jornal Brasileiro de Ginecologia, Rio de Janeiro, v.106, n.6, p.197-204, 1996.

DONANGELO, C.M., TRUGO, N.M.F., KOURY, J.C., BARRETO SILVA, M.I., FREITAS, L.A., FELDHEIM, W., BARTH, C. Iron, zinc, folate and vitamin B12 nutritional status and milk composition of low income Brazilian mothers. European Journal of Clinical Nutrition, London, v.43, n.4, p.253-266, 1989.

DONANGELO, C.M., TRUGO, N.M.F., MELO, G.J.O., GOMES, D.D., Henriques, C. Calcium homeostasis during pregnacy and lactation in primiparous women with sub-adequate calcium intakes. Nutrition Research Reviews, Cambridge, v.16, n.9, p.1631-1640, 1996.
EDWARDS, L.E., DIECKES, W.F., ALTON, J.R., HAKANSON, E.Y. Pregnancy in the massively obese: course, outcome and obesity prognosis of infant. American Journal of Obstetrics and Gynecology, Saint Louis, v.131, n.2, p.479-483, 1978.

ENGSTROM E.M., ANJOS, L.A. Relação entre o estado nutricional materno e sobrepeso nas crianças brasileiras. Revista de Saúde Pública, São Paulo, v.30, n.3, p.233-239, 1996.

FRENTZEN, B.H., IMPERIO, D.L., CRUZ, A.C. Maternal weight gain: effect on infant birth weight among overweight and average - weight low income women. American Journal of Obstetrics and Gynecology, Saint Louis, v.159, n.5, p.1114-1127, 1988.

GUTHRIE, H.A., PICCIANO, M.F. Human nutrition. Saint Louis : MOSBY, 1995. 654p.

KING, J.C., WEININGER, J. Embarazo y lactancia. Washington DC : Organización Panamericana de la Salud, 1991. p.362-368. (OPAS - Publicación Cientifica, 532).

MIKODE, M.S., WHITE, A.A. Dietary assessment of middle income pregnant women during the first, second and third trimesters. Journal of the American Dietetic Association, Chicago, v.94, n.2, p.196-199, 1994.

NATIONAL RESEARCH COUNCIL (USA). Recommended dietary allowances. Washington DC : National Academy Press, 1989. 283p.

NEUHOUSER, L.S.N. Nutrition during pregnancy and lactation. In: MAHAN, L.K., ESCOTT-STUMP, S. Krause's: food, nutrition \& diet therapy. 9.ed. Philadelphia : W.B. Saunders, 1996. p.181-212.

ORGANIZACIÓN MUNDIAL DE LA SALUD. Dieta, nutrición y prevención de enfermidades no transmissibles. Ginebra, 1990. 228p. (Série de Informes Técnicos, 797).

SHOJANIA, A.M. Folic acid and vitamin $B_{12}$ deficiency in pregnancy and neonatal period. Clinics in Perinatology, Philadelphia, v.11, n.2, p.433-459, 1984.

STEVEN-SIMON C., ROGHMANN, K.J., McANARNEY, E.R. Relationship of self-reported prepregnant weight gain during pregnancy to maternal body 
habits and age. Journal of the American Dietetic Association, Chicago, v.92, n.1, p.85-87, 1992.

SUSSER, M. Maternal weight gain, infant birth weight, and diet: causal sequences. American Journal of Clinical Nutrition, Bethesda, v.53, n.3, p.291-307, 1991.

TANAKA, A.C. A importância da associação obesidade e gravidez. Revista de Saúde Pública, São Paulo, v.15, n.1, p.291-307, 1981.

TRUGO, N.M.F., DONANGELO, C.M., SEYFARTH, B.S.P., HENRIQUES, C., ANDRADE, L.P. Folate and iron status of non-anemic women during pregnancy: effect of routine folate and iron supplementation and relation of erytrocyte folate and iron stores. Nutrition Research Reviews, Cambridge, v.16, n.6, p.1267-1276, 1996.

UCHIYAMA, M., SANTANA, R.M., NOVO, N.F., BERTINI, A.M., CAMANO, L. Obesidade e gravidez: análise da idade materna e da paridade. Jornal Brasileiro de Ginecologia, Rio de Janeiro, v.103, n.1, p.193-198, 1993.
VANNUCCHI, H., FREITAS, M.L., SZARFARC, S.C. Prevalência de anemias nutricionais no Brasil. Cadernos de Nutrição, São Paulo, v.4, n.1, p.7-26, 1992.

WEIGEL, M.M., NARVARÉZ, W.M., AMPARO, L., FÉLIX, C., LÓPEZ, P. Prenatal diet, nutrient intake and pregnancy outcome in urban ecuadorian primiparas. Archivos Latinoamericanos de Nutrición, Guatemala, v.41, n.1, p.21-37, 1991.

WORLD HEALTH ORGANIZATION. Physical status: the use and interpretation of anthropometry. Geneva, 1995. 452p. (Techinical Report Series, 854).

YOON, B.K., LEE, J.W., CHOI, D.S., ROH, C.R., LEE, J.H. Changes in biochemical bone markers during pregnancy and puerperium. Journal of Korean Medical Science, Seoul, v.15, n.2, p.189-193, 2000.

Recebido para publicação em e 4 de setembro de 2000 e aceito em 28 de agosto de 2001. 